\title{
Exploring Three Nucleon Forces in Lattice QCD
}

\author{
Takumi Doi (for HAL QCD Collaboration)
}

\author{
Graduate School of Pure and Applied Sciences, University of Tsukuba, Tsukuba 305-8571, Japan
}

\begin{abstract}
We study the three nucleon force in $N_{f}=2$ dynamical clover fermion lattice QCD, utilizing the Nambu-Bethe-Salpeter wave function of the three nucleon system. Since parity-odd two nucleon potentials are not available in lattice QCD at this moment, we develop a new formulation to extract the genuine three nucleon force which requires only the information of parity-even two nucleon potentials. In order to handle the extremely expensive calculation cost, we consider a specific three-dimensional coordinate configuration for the three nucleons. We find that the linear setup is advantageous, where nucleons are aligned linearly with equal spacings. The lattice calculation is performed with $16^{3} \times 32$ configurations at $\beta=1.95, m_{\pi}=1.13 \mathrm{GeV}$ generated by CP-PACS Collaboration, and the result of the three nucleon force in triton channel is presented.
\end{abstract}

Keywords: Lattice QCD, Nuclear Forces, Three Nucleon Force

PACS: 12.38.Gc, 13.75.Cs, 21.30.-x, 21.45.Ff

\section{INTRODUCTION}

Understanding the interaction among nucleons is one of the most important issues in particle and nuclear physics. In particular, the properties of the three nucleon force (TNF) are the forefront of not only particle/nuclear physics but also astro physics. For the binding energies of light nuclei, the two nucleon $(2 \mathrm{~N})$ force alone underestimates the experimental data, and attractive TNF components are required [1]. On the other hand, repulsive TNF components are also necessary in both of symmetric and asymmetric nuclear matters, in order to reproduce the empirical saturation density of nuclei in the former case, and to make the equation of state of asymmetric nuclear matter hard enough to sustain the observed massive neutron star masses in the latter case [2]. Recently, it is argued that TNF is responsible for the anomaly in the drip line and the nontrivial magic number of neutron-rich nuclei [3,4], and could impact on the supernova nucleosynthesis.

Pioneered by Fujita-Miyazawa [5], TNF have been commonly studied from the twopion exchange models with the $\Delta$-excitation. However, since TNF is originated by the fact that a nucleon is not a fundamental particle, it is desirable to study TNF from the fundamental degrees of freedom (DoF), i.e., quarks and gluons. In this report, we carry out first-principle calculations of TNF using the lattice QCD technique.

In the lattice QCD calculation of nuclear forces, it is recently proposed [6] to use the Nambu-Bethe-Salpeter (NBS) wave function so that the potential is faithful to the phase shift by construction. The obtained (parity-even) $2 \mathrm{~N}$ potentials are found to have desirable features, such as attractive well at long and medium distances, and the central repulsive core at short distance [6,7]. It is also examined that the non-locality of the obtained potential is small, and the leading terms in the derivative expansion dominate the potential at low energies [8]. The method has been successfully extended to the hyperon-nucleon (YN) and hyperon-hyperon (YY) interactions $[9,10,11]$ including 
H-dibaryon system [12]. Further applications are given in Refs. [13, 14, 15, 16]. In this report, we discuss the extension of this approach to the three nucleon $(3 \mathrm{~N})$ system, and lattice QCD calculation of TNF in the triton channel is carried out. A preliminary account of this study is also given in Ref. [17].

\section{FORMALISM}

Since the detailed formulation for the $2 \mathrm{~N}$ force is given in Ref. [7], we discuss the extension to the $3 \mathrm{~N}$ system. We consider the equal-time NBS wave function $\psi\left(\overrightarrow{r^{\prime}}, \vec{\rho}\right)$, which can be obtained through the six point correlator,

$$
G_{\alpha \beta \gamma, \alpha^{\prime} \beta^{\prime} \gamma^{\prime}}\left(\overrightarrow{r^{\prime}}, \vec{\rho}, t-t_{0}\right)=\left\langle N_{\alpha}\left(\vec{x}_{1}, t\right) N_{\beta}\left(\vec{x}_{2}, t\right) N_{\gamma}\left(\vec{x}_{3}, t\right) \overline{\left(N_{\alpha^{\prime}}\left(t_{0}\right) N_{\beta^{\prime}}\left(t_{0}\right) N_{\gamma^{\prime}}\left(t_{0}\right)\right)}\right\rangle,
$$

where $\overrightarrow{r^{\prime}} \equiv \vec{x}_{1}-\vec{x}_{2}, \vec{\rho} \equiv \vec{x}_{3}-\left(\vec{x}_{1}+\vec{x}_{2}\right) / 2$ are the Jacobi coordinates, and $N$ denotes either of $p$ or $n$. With the derivative expansion of the potentials, the NBS wave function can be converted to the potentials through the following Schrödinger equation,

$$
\left[-\frac{1}{2 \mu_{r^{\prime}}} \nabla_{r^{\prime}}^{2}-\frac{1}{2 \mu_{\rho}} \nabla_{\rho}^{2}+\sum_{i<j} V_{2 N, i j}\left(\vec{x}_{i j}\right)+V_{T N F}\left(\vec{r}^{\prime}, \vec{\rho}\right)\right] \psi\left(\overrightarrow{r^{\prime}}, \vec{\rho}\right)=E \psi\left(\overrightarrow{r^{\prime}}, \vec{\rho}\right),
$$

where $V_{2 N, i j}\left(\vec{x}_{i j}\right)$ with $\vec{x}_{i j} \equiv \vec{x}_{i}-\vec{x}_{j}$ denotes the potential between $(i, j)$-pair, $V_{T N F}\left(\overrightarrow{r^{\prime}}, \vec{\rho}\right)$ the TNF, $\mu_{r^{\prime}}=m_{N} / 2, \mu_{\rho}=2 m_{N} / 3$ the reduced masses. If we calculate $\psi\left(\overrightarrow{r^{\prime}}, \vec{\rho}\right)$ for all $\overrightarrow{r^{\prime}}$ and $\vec{\rho}$, and if all $V_{2 N, i j}\left(\vec{x}_{i j}\right)$ are available by (separate) lattice calculations for the genuine $2 \mathrm{~N}$ system, we can extract $V_{T N F}\left(\overrightarrow{r^{\prime}}, \vec{\rho}\right)$ through Eq. (2).

In practice, however, the calculation cost of $\psi\left(\overrightarrow{r^{\prime}}, \vec{\rho}\right)$ is prohibitively expensive. Note that the number of diagrams generated by Wick contractions tends to diverge with a factor of $N_{u} ! \times N_{d} !\left(N_{u, d}\right.$ are numbers of u,d quarks in the system). We develop several techniques to reduce the calculation cost, e.g., we take advantage of symmetries (such as isospin symmetry), and employ the non-relativistic limit for the source nucleon operator. Similar techniques are (independently) developed in the calculations of binding energies of triton/helium nuclei [18]. Yet, the calculation cost remains quite expensive, so we consider Eq. (2) with fixed three-dimensional (3D) coordinates of $\overrightarrow{r^{\prime}}$ and $\vec{\rho}$.

To proceed, we find that the linear setup with $\vec{\rho}=\overrightarrow{0}$ is advantageous, where three nucleons are aligned linearly with equal spacings of $r \equiv|\vec{r}| \equiv 1 / 2\left|\overrightarrow{r^{\prime}}\right|$. In fact, because of $\vec{\rho}=\overrightarrow{0}$, the third nucleon is attached to $(1,2)$-nucleon pair with only S-wave. Considering the total $3 \mathrm{~N}$ quantum numbers of $I=1 / 2, J^{P}=1 / 2^{+}$(triton channel), the wave function can be completely spanned by only three bases, which can be labeled by the quantum numbers of $(1,2)$-pair as ${ }^{1} S_{0},{ }^{3} S_{1},{ }^{3} D_{1}$. Therefore, the Schrödinger equation leads to the $3 \times 3$ coupled channel equations with the bases of $\psi_{1} S_{0}, \psi_{3} S_{1}, \psi_{3} D_{1}$. The reduction of the dimension of bases is expected to improve the $\mathrm{S} / \mathrm{N}$ as well.

Even when we calculate with the fixed 3D-configuration (with the linear setup or whatever), the identification of genuine TNF remains nontrivial. This is because the parity-odd $2 \mathrm{~N}$ potentials are not available in lattice QCD at this moment, and we cannot subtract the contribution of $V_{2 N}$ unambiguously. Note that although the total parity of the 
$3 \mathrm{~N}$ system can be projected, a $2 \mathrm{~N}$-pair inside the $3 \mathrm{~N}$ system could be either of positive or negative parity. The familiar procedure of partial wave expansion cannot be performed here, since we calculate only fixed 3D-configurations to reduce the calculation cost.

To overcome this problem, we find that the following channel in the triton is useful,

$$
\psi_{S} \equiv \frac{1}{\sqrt{6}}\left[-p_{\uparrow} n_{\uparrow} n_{\downarrow}+p_{\uparrow} n_{\downarrow} n_{\uparrow}-n_{\uparrow} n_{\downarrow} p_{\uparrow}+n_{\downarrow} n_{\uparrow} p_{\uparrow}+n_{\uparrow} p_{\uparrow} n_{\downarrow}-n_{\downarrow} p_{\uparrow} n_{\uparrow}\right] .
$$

This wave function itself has been well known, but the point here is that it is antisymmetric in spin/isospin spaces for any $2 \mathrm{~N}$-pair. Combined with the Pauli-principle, it is automatically guaranteed that any $2 \mathrm{~N}$-pair couples with even parity only. Therefore, we can extract TNF unambiguously in this channel, without the information of parityodd $2 \mathrm{~N}$ potentials. Note that no assumption on the choice of $3 \mathrm{D}$-configuration is imposed in this argument, and we can take advantage of this feature in future TNF calculations with 3D-configurations other than the linear setup.

\section{LATTICE QCD RESULTS}

We employ $N_{f}=2$ dynamical clover fermion configurations generated by CP-PACS Collaboration [19]. We use 598 configurations at $\beta=1.95$ with the lattice size of $L^{3} \times T=16^{3} \times 32$, which corresponds to $(2.5 \mathrm{fm})^{3}$ box in physical spacial size with the lattice spacing of $a^{-1}=1.269 \mathrm{GeV}$. We calculate at the hopping parameter of $u, d$ quarks $\kappa_{u d}=0.13750$, which corresponds to $m_{\pi}=1.13 \mathrm{GeV}, m_{N}=2.15 \mathrm{GeV}$. We use the wall quark source with Coulomb gauge fixing. In order to enhance the statistics, we perform the calculation for sources on 16 time slices for each configuration.

We perform the calculation with the linear setup at ten physical points of the distance $r$. Since we have only one channel (Eq. (3)) which is free from parity-odd $2 \mathrm{~N}$ potentials, we can determine one type of TNF. In this report, we consider the scalar/isoscalar type TNF. In the Urbana IX model [20], which is an often-used phenomenological TNF, TNF consists of two parts, one is the two-pion exchange TNF and the other is phenomenologically introduced scalar/isoscalar repulsive TNF. Since the two-pion exchange TNF is expected to be suppressed in the current lattice setup of $m_{\pi}=1.13$ $\mathrm{GeV}$, it is reasonable to assume the scalar/isoscalar nature for TNF here.

In Fig. 1 (left), we plot each wave function of $\psi_{S}=\left(-\psi^{1} S_{0}+\psi_{3} S_{1}\right) / \sqrt{2}, \psi_{M} \equiv$ $\left(\psi_{S_{0}}+\psi_{3} S_{1}\right) / \sqrt{2}$ and $\psi_{3} D_{1}$ in the triton channel at $t-t_{0}=8$. We observe that $\psi_{S}$ dominates the wave function. This is because $\psi_{S}$ contains the component for which all three nucleons are in $S$-wave. By solving the $3 \times 3$ coupled channels and subtracting the $V_{2 N}$ from the total potentials, we determine TNF. In Fig. 1 (right), we plot the results for the scalar/isoscalar TNF. Here, the $r$-independent shift by energies is not included, and thus about $\mathscr{O}(10) \mathrm{MeV}$ systematic error is understood. At the long distance region of $r$, TNF is small as is expected. At the short distance region, we observe the indication of repulsive TNF. Recalling that the repulsive short-range TNF is phenomenologically required in high density matter, this is very encouraging result. Of course, we note that further researches are necessary, e.g., the examination of the discretization error and the dependence on the quark masses. Studies along this line is currently underway. 

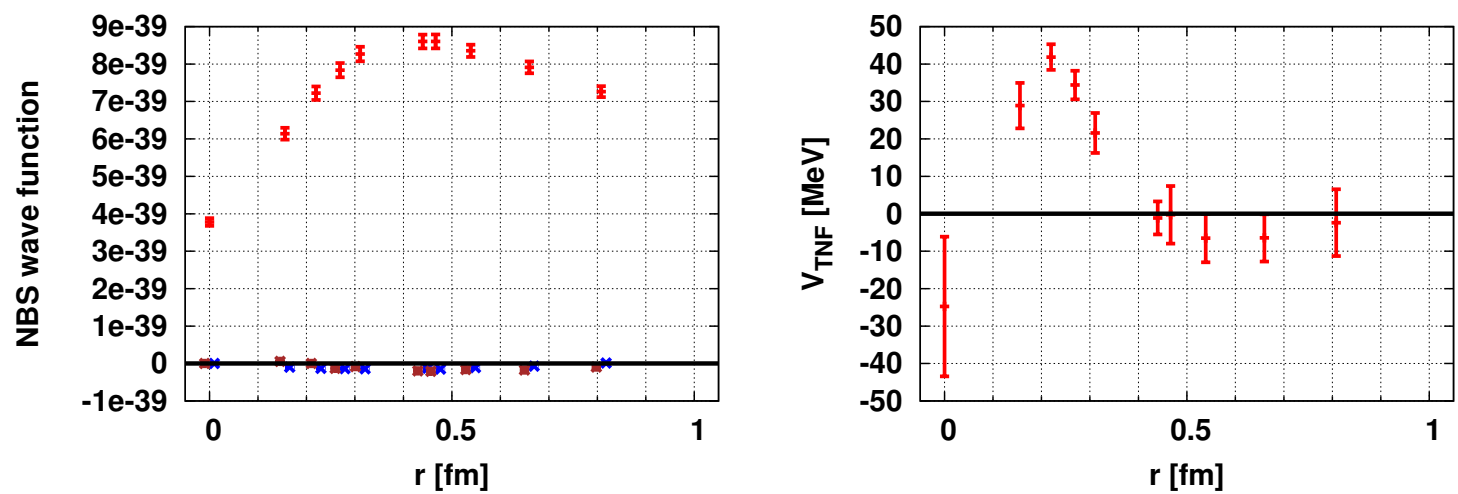

FIGURE 1. (color online). (Left) The wave function with linear setup in the triton channel. Red, blue, brown points correspond to $\psi_{S}, \psi_{M}, \psi^{3} D_{1}$, respectively. (Right) The scalar/isoscalar TNF in the triton channel, plotted against the distance $r$ in the linear setup.

We thank authors and maintainers of CPS++[21], and CP-PACS Collaboration and ILDG/JLDG [22] for providing gauge configurations. The numerical calculations have been performed on T2K at University of Tsukuba (09a-11, 10a-19) and Blue Gene/L at KEK (09-23, 09/10-24). This research is supported in part by MEXT Grant-in-Aid, Scientific Research on Innovative Areas (20105001, 20105003, 21105515). T. D. is supported in part by Grant-in-Aid for JSPS Fellows $21 \cdot 5985$.

\section{REFERENCES}

1. S. C. Pieper, Riv. Nuovo Cim. 31, 709 (2008), [arXiv:0711.1500 [nucl-th]].

2. A. Akmal, V. R. Pandharipande and D. G. Ravenhall, Phys. Rev. C58, 1804 (1998).

3. T. Otsuka, T. Suzuki, J. D. Holt, A. Schwenk and Y. Akaishi, Phys. Rev. Lett. 105, 032501 (2010).

4. J. D. Holt, T. Otsuka, A. Schwenk and T. Suzuki, [arXiv:1009.5984 [nucl-th]].

5. J. Fujita and H. Miyazawa, Prog. Theor. Phys. 17, 360 (1957).

6. N. Ishii, S. Aoki and T. Hatsuda, Phys. Rev. Lett. 99, 022001 (2007).

7. S. Aoki, T. Hatsuda and N. Ishii, Prog. Theor. Phys. 123, 89 (2010).

8. K. Murano, N. Ishii, S. Aoki and T. Hatsuda, Prog. Theor. Phys. in press, [arXiv:1103.0619 [hep-lat]].

9. H. Nemura, N. Ishii, S. Aoki and T. Hatsuda, Phys. Lett. B673, 136 (2009).

10. T. Inoue et al. [HAL QCD Collab.], Prog. Theor. Phys. 124, 591 (2010).

11. K. Sasaki [HAL QCD Collab.], PoS LATTICE2010, 157 (2010), [arXiv:1012.5685 [hep-lat]].

12. T. Inoue et al. [HAL QCD Collab.], Phys. Rev. Lett. 106, 162002 (2011).

13. Y. Ikeda et al., [arXiv:1002.2309 [hep-lat]].

14. Y. Ikeda and H. Iida, [arXiv:1102.2097 [hep-lat]].

15. T. T. Takahashi and Y. Kanada-En'yo, Phys. Rev. D82, 094506 (2010).

16. T. Kawanai and S. Sasaki, Phys. Rev. D82, 091501 (2010).

17. T. Doi [HAL QCD Collab.], PoS LATTICE2010, 136 (2010), [arXiv:1011.0657 [hep-lat]].

18. T. Yamazaki, Y. Kuramashi and A. Ukawa, [PACS-CS Collab.], Phys. Rev. D81, 111504 (2010).

19. A. Ali Khan et al. [CP-PACS Collab.], Phys. Rev. D 65, 054505 (2002) [E: D 67, 059901 (2003)].

20. B. S. Pudliner, V. R. Pandharipande, J. Carlson and R. B. Wiringa, Phys. Rev. Lett. 74, 4396 (1995).

21. Columbia Physics System (CPS), http: / / qcdoc . phys . columbia . edu/cps . html

22. http://www.lqcd.org/ildg, http://www.jldg.org 